NASA Technical Memorandum 106830

\title{
The Effect of Temperature on the Radiative Performance of Ho-YAG Thin Film. Selective Emitters
}

Roland A. Lowe

Kent State University

Kent, Ohio

and

Donald L. Chubb and Brian S. Good

Lewis Research Center

Cleveland, Ohio

Prepared for the

First World Conference on Photovoltaic Energy Conversion cosponsored by IEEE, PVSEC-Japan, and PVSEC-Europe Waikoloa; Hawaii, December 5-9, 1994

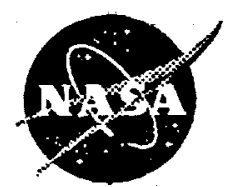

National Aeronautics and Space Administration
(NASA-TM-106830) THE EFFECT OF TEMPERATURE ON THE RADIATIVE PERFORMANCE OF HO-YAG THIN FILM SELECTIVE EMITTERS (NASA. LEWIS Research (enter) $6 \mathrm{p}$
N95-18587

Unclas

G3/44 0035862 


$$
\begin{array}{lll}
\cdots & \ldots & -
\end{array}-\ldots
$$




\title{
THE EFFECT OF TEMPERATURE ON THE RADIATIVE PERFORMANCE OF HO-YAG THIN FILM SELECTIVE EMITTERS
}

\author{
Roland A. Lowe*, Donald L. Chubb ${ }^{+}$and Brian S. Good ${ }^{+}$ \\ * Kent State University, Kent, Ohio 44242 \\ + NASA Lewis Research Center, Cleveland, Ohio 44135
}

\begin{abstract}
In this paper we present the emitter efficiency results for the thin film 25\% Ho YAG (Yttrium Aluminum Garnet, $\mathrm{Y}_{3} \mathrm{Al}_{5} \mathrm{O}_{12}$ ) selective emitter from 1000-1700 $\mathrm{K}$ with a platinum substrate. Spectral emittance and emissive power measurements were made $(1.2<\lambda<3.2 \mu \mathrm{m})$ and used to calculate the radiative efficlency. The radiative efficlency and power density of rare earth doped selective emitters are strongly dependent on temperature and experimental results indicate an optimum temperature (1650 K for Ho YAG) for TPV applications.
\end{abstract}

\section{INTRODUCTION}

Efficient thermophotovoltaic (TPV) energy conversion is strongly dependent on emitter performance[1]. The most promising solid state selective emitters are compounds containing elements in the Lanthanide Series (rare earths). Nelson and Parent[2,3,4] using the rare earth oxide Welsbach mantle emitter have been the most successful. A new class of rare earth solid state selective emitters, the rare earth gamets $[5,6]$, has the potential for being efficient, as well as, more durable than the mantle type emitter. In this paper we present experimental results for the radiative efficiency of a YAG (Yttrium Aluminum Garnet, $\mathrm{Y}_{3} \mathrm{Al}_{5} \mathrm{O}_{12}$ ) thin film selective emitter doped with holmium (Ho) for a temperature range of 1000 to $1700 \mathrm{~K}$ and compare it to the theoretical results predicted using[7].

\section{RARE EARTH GARNET SELECTIVE EMITTER}

The atomic structure of the doubly and triply charged rare earth ions such as $\mathrm{Yb}, \mathrm{Er}, \mathrm{Ho}, \mathrm{Tm}, \mathrm{Dy}$, and Nd accounts for their unique spectral emission characteristics. The orbits of the valence 4 electrons, whose electronic transitions determine the spectral emission properties, lie inside the $5 s$ and $5 p$ electron orbits. The $5 s$ and $5 p$ electrons "shield" the $4 f$ valence electrons from the surrounding ions in the crystal. As a result, the rare earth ions in the solid state have radiative characteristics much like an isolated atom in a gas and emit in relatively narrow bands rather than in a continuum as do most solids.

As already mentioned, the first successful rare earth selective emitters were the rare earth oxides. Recently rare earth doped YAG has shown excellent emissive properties[5]. Doping limits, dependent on the particular ion and crystal host may be as high as 100 at. $\%$ (complete substitution of the yttrium ion with the rare earth ion). These materials have high metting points (1940 C for undoped YAG) making them excellent candidates for a high temperature thin film selective emitter. For most of the rare earths the emission spectra is composed of emission bands in the near infrared $(0.9<\lambda<3.0 \mu \mathrm{m})$ with relatively low emission outside these bands. For thermally stimulated emission, where the density of exclted states is govemed by Boltzmann equilibrium, the emission spectrum is dominated by a single emission band from the deexcitation(spontaneous emission) of the 1st level to the ground state. Since YAG can be doped with more than a single rare earth it is possible to make a thin film selective emitter with multiple emission bands. As a result, greater power density over a wider wavelength interval can be achieved than with a single rare earth dopant.[6]

The efficiency, $\eta_{E}$, of a selective emitter is defined as

$$
\eta_{E}=\frac{a_{b}}{Q_{T}}
$$

where $Q_{b}$ is the emitted power in the emission band and $Q_{T}$ is the total emitted power.

$$
\begin{aligned}
& a_{b}=\int_{\lambda_{u}}^{\lambda_{1}} q_{\lambda} d \lambda \\
& a_{T}=\int_{0}^{\infty} q_{\lambda} d \lambda
\end{aligned}
$$


where $q_{\lambda}$ is the spectral emissive power of the emitter, $\lambda_{4}$ is the short wavelength limit for the emission band and $\lambda_{1}$ is the long wavelength limit for the emission band. In the experiment to be described in the next section $q_{\lambda}$ is measured with a spectroradiometer and $Q_{b}$ and $Q_{\text {r are }}$ measured with a pyroelectric radiometer.

\section{EXPERIMENTAL PROCEDURE}

The emissivity of a material is usually thought of as a property of an isothermal surface. However, for the thin film rare-earth YAG emitter emission from throughout the film contributes to the spectral emittance. Since significant temperature differences $(210-260 \mathrm{~K})$ exist between the front and back emitter surfaces the effect of the temperature gradient on the radiative performance of the the thin film selective emitter is investigated. For the theoretical analysis the "emitter temperature" is defined by the substrate temperature, $T_{8}$ and the temperature gradient, $\Delta T=T_{s}-T_{\text {Frort. }}$ The average of the front and back surface temperatures, $T_{\mathrm{avg}}=\left(T_{\mathrm{s}}+T_{\text {Front }}\right) / 2$, in the center of the sample is used to calculate spectral emittance from the spectroradiometer intensity measurements. A radial temperature gradient of $5 \mathrm{~K}$ was also measured on the sample in the field of view of the pyroelectric radiometer. This variation, small in comparison to the front-back temperature gradient and other sources of experimental error, was neglected in the emittance calculations. Temperature measurements, made with type $R$ thermocouples, had an accuracy of $+/$ 6K. Total power and power in the Ho YAG emission band was measured with a Laser Precision Rk-5100 radiometer calbrated to an NBS traceable standard. Normal spectral emittance, $\varepsilon_{\lambda}$, measurements were made with a spectroradiometer constructed from a $1 / 8$ meter monochrometer, a temperature controlled PbS detector, and an $800 \mathrm{~Hz}$ chopper calibrated with a $1273 \mathrm{~K}$ blackbody reference. Energy in the emission band, $\boldsymbol{Q}_{\text {b }}$, is measured with the spectroradiometer and calculated using eq. 2. Normal spectral emittance measurement accuracy and repeatability with the blackbody reference at $1273 \mathrm{~K}$ was ypically within $2 \%$ from $1.2<\lambda<3.2 \mu \mathrm{m}$. A series of precision optical pinholes was used to limit the field of view at the specimen surface for both the spectroradiometer and the pyroelectric radiometer. Custom software developed with LabView for the Macintosh was used to calculate spectral emittance and emissive power, and emitted power/wavelength-interval from the intensity measurements.

Specimens were cut from Czochralski grown crystals and polished on both sides with $1 \mu \mathrm{m}$ diamond abrasive. Platinum foll was placed behind the samples as a low emittance substrate. The configuration shown in fig. 1 was used to eliminate reflected radiation from the fumace interior, a near blackbody radiator, from reaching

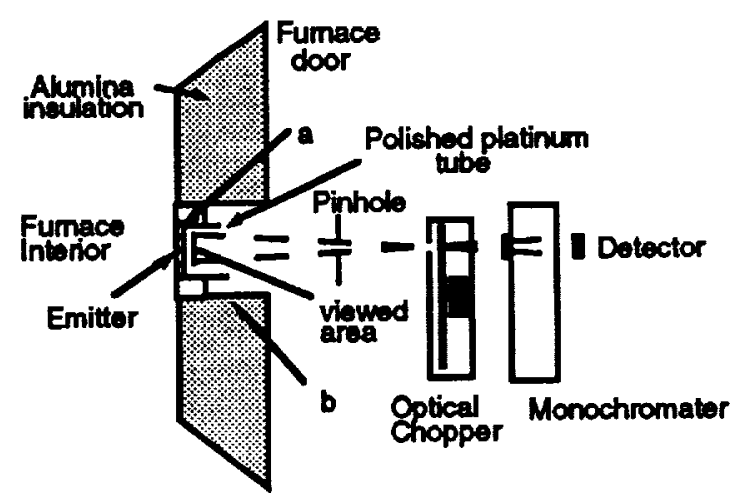

FGURE 1. Schematic diagram of experimental setup

the detector. Two sources of measurement error were identified. Alumina insulation, a nearty perfect diffuse reflector, reflects radiation originating from the perimeter of the sample (a), which is at a higher temperature than the viewed area, to the surface of the sample in the field of view of the detector. Also, emission from the hot alumina (b) is reflected directly from the viewed area to the detector. A polished (specular) platinum tube was inserted in the hole through the insulation near the specimen to reduce the measurement error from these combined effects.

Comparison of $\varepsilon_{\lambda}$ measurements and data from [8] for polished Pt foil show the error in $\varepsilon_{\lambda}$ due to reflected radiation is dependent on the specimen temperature. Raising the specimen temperature increases the intensity of the background radiation and shifts the energy distribution to shorter wavelengths. At 1640K the increase in $E_{\lambda}$ due to reflected radiation and temperature measurement error is +0.029 $1.5 \mu \mathrm{m},+0.046 \odot 2.0 \mu \mathrm{m}$, and $+0.049-3.0 \mu \mathrm{m}$. This will be the maximum measurement error for materials with high spectral reflectance $(-80 \%)$ and low emittance such as platinum. However, in the Ho YAG specimen, reflectance in the emission band is less (20-70\% for Ho-YAG) and the actual measurement error will be lower than the error for platinum.

\section{EXPERIMENTAL RESULTS}

Thin film selective emitter performance is dependent on factors such as emitter thickness and temperature, substrate emittance and rare earth dopant type and concentration[9]. For maximum emitter performance it is essential that these parameters be optimized. The experimental results presented here for the Ho YAG thin film selective emitter do not include efforts to maximize performance through optimization of these parameters. The thickness and doping level of the test specimen reflects the commercial availability of material developed for solid state lasers. Even without optimization results indicate that rare earth thin film selective emitters have potential as elements in a high efficiency TPV system. 
A $1.1 \mathrm{~mm}$ thick, $30 \mathrm{~mm}$ dia. section of $25 \%$ HoYAG with a Pt foil substrate was used to investigate the effect of emitter temperature on radiative efficiency. Normal epectral emittance, Ex, measurements were made from $1.2<\lambda<3.2 \mu \mathrm{m}$ for a series of temperatures ranging from $1000-1700 \mathrm{~K}$. The radiative efficiency, $\eta_{E}$, was calculated using eqs. 1,2,3 with $\lambda_{v}=1.8 \mu \mathrm{m}$ and $\lambda_{1}=2.2$ $\mu \mathrm{m}$ defining the emission band interval used to calculate

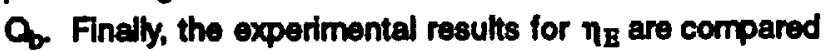
to the theoretical results for $\eta_{\mathrm{E}}$ calculated using [7].

Figure 2 shows the normal spectral emittance in the errission band of $25 \%$ Ho YAG $\left(5 I_{7}-5 l_{8} \odot 2.0 \mu \mathrm{m}\right)$ at

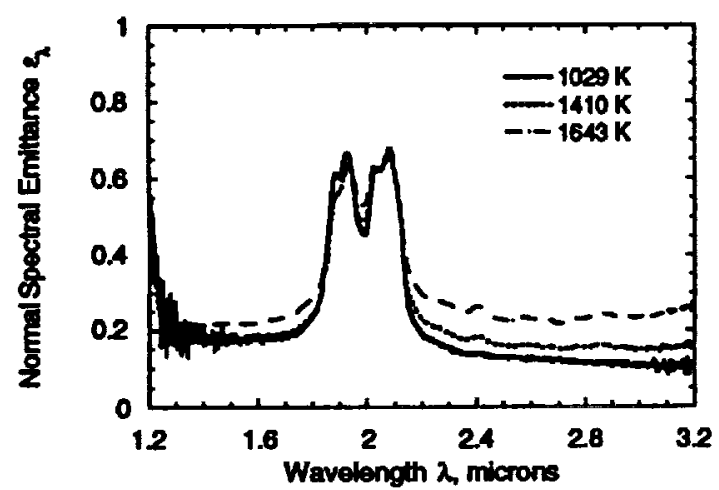

Fig 2 Normal spectral emittance of $25 \%$ Ho-YAG, thickness $=1.1 \mathrm{r}$ at 1029,1410 and $1643 \mathrm{~K}$

three values for $T_{\text {avg, }} 1029,1410$ and $1643 \mathrm{~K}$, from $1.2<\lambda$ $<3.2 \mu \mathrm{m}$. The spectral distribution of the emitted radiation can be divided into two regions. Relatively strong emission is present from $1.8<\lambda<2.2 \mu \mathrm{m}$, also the wavelength interval used to calculate $O_{b}$, at the characteristic emission band. Outside the emission band the extinction coefficient for rare earth YAG is relatively low $\left(-0.1 \mathrm{~cm}^{-1}, 0.3<\lambda<5.0 \mu \mathrm{m}\right)$ and the emission in this region will be dominated by the platinum substrate.

Several important features can be discerned from this figure. First, except for variations caused by thermal line broadening, the spectral emittance in the emission band is relatively temperature invariant. Second, since the optical and radiative properties of this material in the emission band is dominated by the electronic transitions of the substitutional rare earth impurity this result is expected. Third, the increase in $\varepsilon_{\lambda}$ outside the emission band is greater than the expected increase in the spectral emittance of $P I$ from 1000-1700 K.

The rapid increase of $E_{\lambda}$ outside the emission band cannot be attributed to an increase in the substrate omittance, $\varepsilon_{\lambda}$ for $P t$ is actually less at $1633 \mathrm{~K}$ than $1410 \mathrm{~K}$ for $\lambda=2.0 \mu \mathrm{m}[8]$. Data for the high temperature spectral emittance of oxide materials similar (low $\alpha_{\lambda}$ ) to YAG such as sapphire. ALON, and spinel show that the spectral emittance of these materials is very low, $\varepsilon_{2}<0.05$, and remains essentially constant for temperatures up to $\mathbf{2 0 0 0}$ $K$. As previously mentioned, for a given $\lambda$, the magnitude of reflected background radiation and the error in $E_{2}$ increases with $T_{\text {avg. In addition, the magnitude of this }}$ error will be greater for long wavelengths. This is confirmed in fig 2. l.e. as $T_{\text {avg }}$ increases, the rate of increase in $\varepsilon_{\lambda}$ is greater below the emission band than above the emission band. This resulh is opposite to the expected (measured) results for $E_{2}$ vs $T_{8}$ for Pt[8] The error in $\varepsilon_{\lambda}$ due to background radiation is significantly reduced from $1.8<\lambda<2.2$ um due to the relatively strong absorption in the emission band, also shown in fig. 2. It is important to note that the emission from YAG, however small, is effectively doubled by the reflective substrate and adds to the magnitude of $\varepsilon_{2}$ at all wavelengths Additional measurements are planned to investigate the high temperature (up to $2000 \mathrm{~K}$ ) spectral emittance of YAG without a substrate.

Figure 3 shows the theoretical and experimental

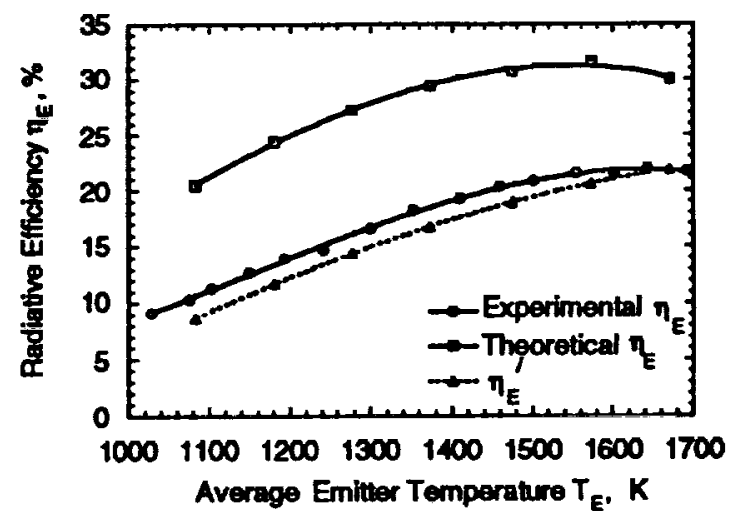

Fig. $3 \eta_{E}$ vs $T_{E}$ for $25 \%$ Ho-YAG, thickness $=1.1 \mathrm{~mm}$, from 1000 to $1700 \mathrm{~K}$

results for the radiative efficiency, $\eta_{E}$. The experimental values are calculated using measured values for $Q_{b}$ and $Q_{T}$ in eq. 1. To calculate theoretical values for $\eta_{G}$ the experimental values of $T_{3}, \Delta T, \varepsilon_{3}$, and the spectral extinction coefficient, $\alpha_{\lambda}$ for Ho-YAG previously reported in [5] are used in the analysis presented in [7].

The theoretical and experimental results for the temperature at which the maximum radiative efficiency occurs, $1650 \mathrm{~K}$ (experimental) and $1550 \mathrm{~K}$ (theoretical). are nearly identical however, the maximum value for $\eta_{E}$ at these temperatures differs by a factor of approximately 1.5. Constraints in the theoretical model require that $\varepsilon_{\mathrm{s}}$ of the substrate and $\alpha_{\lambda}$ outside the emission band be specified as constants. For the theoretical calculation of 
गE the following values were used for $\varepsilon_{3} \varepsilon_{s}=0.25(0<\lambda<$ $1.8 \mu \mathrm{m}), \varepsilon_{8}=0.19(1.8<\lambda<2.2 \mu \mathrm{m})$ and $\varepsilon_{3}=0.15(2.2<$ $\lambda<\omega \mu m)$. Actual measurements show that for Ho YAG and other ahuminum gamots $\alpha_{\lambda}$ increases rapldy for $\lambda>$ $5.0 \mathrm{\mu m}$ and remains high through the far IR. As a resulh $\varepsilon_{\lambda}$ for YAG is aleo expected to be high, -0.95 for $5.0<\lambda<$ $10.0 \mu \mathrm{m}[10]$, and the additional radiation emitted at these wavelengthe will increase the magnitude of $\mathrm{C}_{\mathrm{T}}$ and reduce the experimental value for $\eta_{E}$ for a given $T_{s}$. For this analysls, the contribution of the long wavelength radiation from YAG, $q_{\lambda>5.0}$ is approximated using the Planck equation with the experimental values for $T_{\text {avg }}$ and $e_{\lambda}=$ $0.8,0.95$ (expected emittance for YAG) - 0.15(theoretical value for $\varepsilon_{\text {) }}$, for $5.0 \mu \mathrm{m}<\lambda<\infty$. This result is used to modify the theoretical result for $\alpha_{T}$ and $\eta_{E}$ where

$$
\begin{aligned}
& Q_{T^{\prime}}=Q_{T}+Q_{\lambda>5.0} \\
& \eta_{E^{\prime}}=Q_{\mathrm{b}} / O_{T}{ }^{\prime}
\end{aligned}
$$

The effect on the emission from YAG at $\lambda>5.0 \mu \mathrm{m}$ on the theoretical values values for $\eta_{E}$, represented by $\eta_{E}$, is also shown in fig 4 . The effect of $q_{\lambda>5.0}$ on $Q_{T}$ will be greater for lower values of $T_{3}$ since the amount of radiation emitted below $\lambda=5.0 \mu \mathrm{m}$ decreases proportionately as the temperature increases. Results for $\eta_{E}$ are in good agreement with experimental values for $\eta_{E}$ and suggest that difference in temperature where the maximum theoretical and experimental values of $\eta_{E}$ occurs is due to Q $>5.0$.

As mentioned earlier, the emitter is not optimized with regard to thickness, Ho concentration, and substrate emittance. Using a substrate with a lower $\varepsilon_{s}$ such as hodium will improve $\eta_{E}$. Also, theory[11] indicates the optimum thickness for $25 \%$ Ho YAG is $-0.6 \mathrm{~mm}$, considerably less than the $1.1 \mathrm{~mm}$ thick sample used for this experiment.

\section{CONCLUSION}

The first measured results for the effect of temperature on the radiative efficiency of rare earth doped thin film selective emitters confirms there is an optimum temperature for maximum emitter efficiency. Accounting for the additional long wavelength radiation $(\lambda>5.0 \mu \mathrm{m})$ from the host material(YAG), the experimental and previously reported theoretical results for an Ho doped selective emitter are in good agreement.

\section{REFERENCES}

(1) Chubb D.L., "Reappraisal of Solid Selective Emitters", in Proceedings of the 21 st Photovoltaic Speclallsts Conference IEEE, Now York 1990 pp 1326-1342, also NASA TM108290, 1890

[2] Nelson R.E., in Proceedings of the send Intemational Power Sources Sympostum, Electrochemical Society, Pennington, N, 1986 pp 95-101

[3] Parent C.R. and Nelson R.E., in Proceodings of the Twenty First Intersociety Energy Conversion Engineering Conference, American Chemical Society, Washington DC, 1986 vol. 2, pp 1314-1317

[4] Nelson R.E. and lles P.A. "Space Solar Power for Orbital and Lunar Missions", ASME/ASESSOLTECH Joint Solar Energy Conference, 1903

[5] Lowe R.A., Chubb D.L., Farmor S.C. and Good B.S. "Rare Earth Gamet Selective Emitter" Appl Phys. Letters, 64, 3551 (1994)

[6] Lowe R.A., Chubb D.L, and Good B.S."Radiative Performance of Er and Ho YAG Thin Film Selective Emitters", in First NREL Conference on Thermophotovoltak Generation of Electriclty, American Instifute of Physics, ANP Press, Woocbury NY, 1994 [7] Good B.S., Chubb D.L and Lowe R.A.,TemperatureDependent Eficiency Calculations of a Thin Fim Selective Emitter", in First NREL Conference on Thermophotovoltaic Generation of Electricity. American institute of Physics, ANP Press, Woodbury NY, 1994

[8] Touloukian Y.S. and Ho C.Y.. Thermophysical Properties of Matter vol. 7 Thermal Radiative Properties Metallic Elements \& Alloys", (IFUPlenum, New York, 1970, [9] Chubb D.L. and Lowe R.A., Thin Film Selective Emitter", J. App/ Phys, 74, (1993) pp. 529 and 537

[10] Sova R.M.,Linevsky M.J.,Thomas M.E., and Mark F.F.,"High-Temperature Optical Properties of Oxide Ceramics", Johns Hopkins APL Technical Digest, 13, Pp 375-376, (1992)

[11] Chubb D.L., Lowe R.A., and Good B.S. "Emittance Theory for a Thin Film Selective Emitter", in First NREL Conference on Thermophotovoltaic Generation of Electricity, American Institute of Physics, AlP Press, Woodbury NY, 1994 


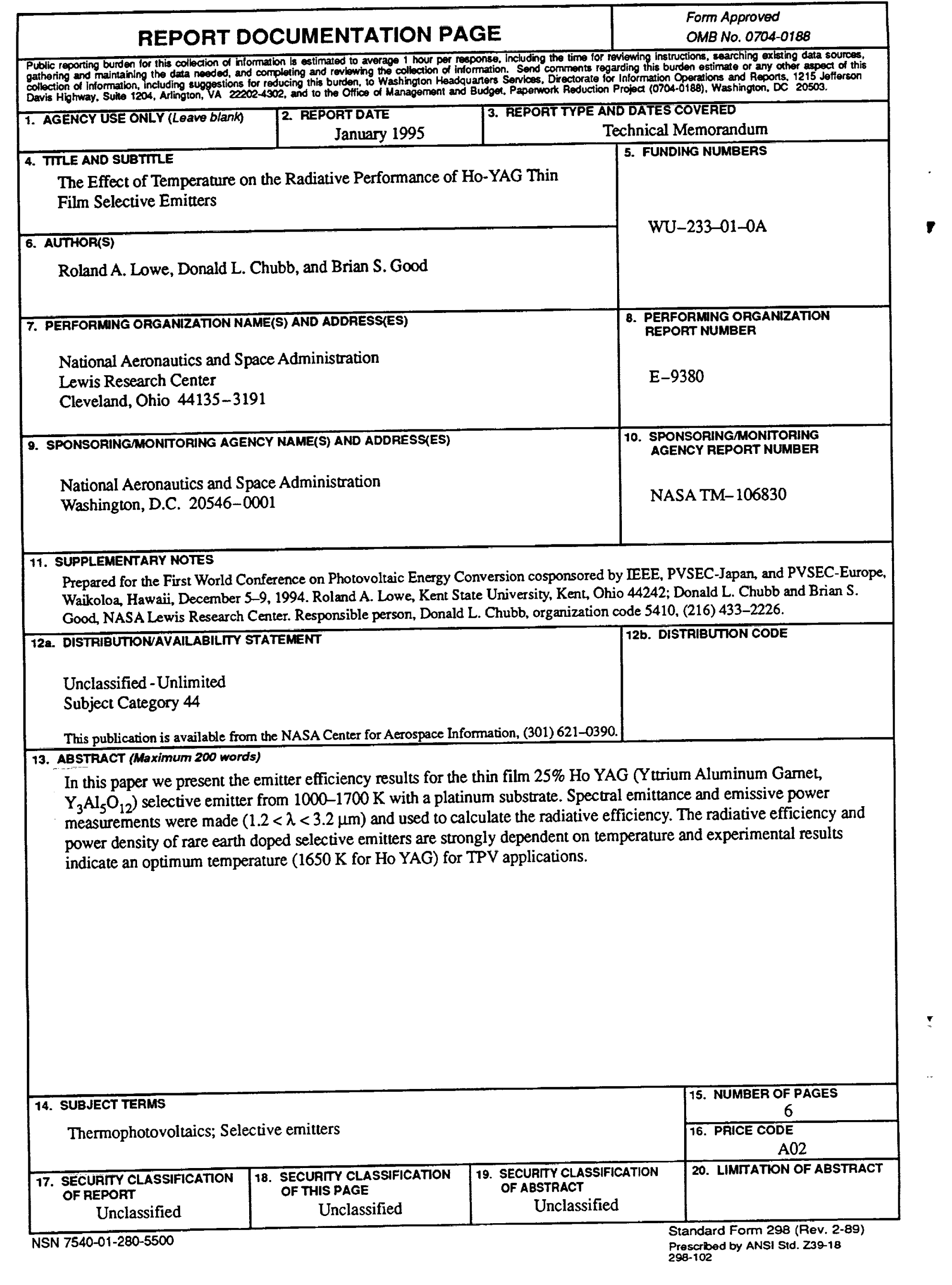

